\title{
"OGNUNO HA BISOGNO DI UN TANA IN CUI LECCARSI LE FERITE: È UN FONDAMENTALE DIRITTO DELL'UOMO”. L'ANALISI DEL DISAGIO ESISTENZIALE ATTRAVERSO LA RAPPRESENTAZIONE DELL'AMBIENTE URBANO NELLA RACCOLTA DI RACCONTI BOLOGNA D'AUTORE A CURA DI GABRIELLA KURUVILLA ${ }^{1}$
}

\author{
Nikica Mihaljević
}

\begin{abstract}
“Ognuno ha bisogno di una tana in cui leccarsi le ferite: è un fondamentale diritto dell'uomo". La rappresentazione del disagio esistenziale attraverso l'ambiente urbano nella raccolta di racconti Bologna d'autore a cura di Gabriella Kuruvilla

Riassunto: Nel presente intervento l'obiettivo è, analizzando l'ambiente urbano in quattro racconti della raccolta Bologna d'autore (2016) curata da Gabriella Kuruvilla, esaminare ed approfondire la crisi d'identità dell'uomo contemporaneo. Nell'analisi si parte dall'ipotesi che lo spazio urbano influenzi e transformi l'identità dei protagonisti e viceversa, nonché determini i rapporti degli individui con l'ambiente circostante. Ci serviamo della metodologia proposta da Hana Wirth-Nesher in City Codes. Reading the Modern Urban Novel, in cui si distinguono quattro possibili tipi dell'ambiente urbano: quello "naturale", costruito, umano e verbale. Lo scopo è scoprire le interrelazioni tra l'individuo e l'ambiente urbano e le conseguenze di esse.

Parole chiave: letteratura italiana contemporanea, ambiente urbano, elemento spaziale, crisi d'identità, disagio esistenziale.
\end{abstract}

"Everyone needs a den in which to lick their wounds: it is a fundamental human right". The Representation of Existential Distress through the Urban Environment in the Short Stories Collection Bologna d'autore edited by Gabriella Kuruvilla

Abstract: In the present article the objective is to examine and deepen the identity crisis of the contemporary man by analyzing the urban environment in four short stories from Bologna d'autore (2016) edited by Gabriella Kuruvilla. In the analysis we start from the hypothesis that the urban space influences and transforms the identity of the characters and vice versa, as well as determines the relationships of individuals with the surrounding environment. We use the methodology proposed by Hana Wirth-Nesher in City Codes. Reading the Modern Urban Novel, in which four possible types of urban environment are distinguished: the "natural", built, human, and verbal type. The aim is to discover the interrelationships between the individual and the urban environment and the consequences of them. Key words: Italian contemporary literature, urban environment, spatial element, identity crisis, existential distress.

\section{Introduzione}

La letteratura contemporanea rianalizza e ridefinisce l'elemento spaziale, in particolare le rappresentazioni e i limiti dello spazio urbano, oggi forse come mai prima nella tradizione

\footnotetext{
${ }^{1}$ Fecha de recepción: 04/11/2019.

Fecha de aceptación: 28/11/2019.

${ }^{2}$ Professoressa Associata, Dipartimento di Italianistica, Università di Spalato, Croazia; $\bowtie$ nikica@ffst.hr.
} 
letteraria e proprio in seguito agli avvenimenti storici, politici, sociali, culturali e altri che hanno contribuito a rendere, come nella società così anche nella letteratura contemporanea, più importante il ruolo dello spazio rispetto a quello del tempo. D’una parte, lo spazio urbano rispecchia quello che avviene ai protagonisti e cambia con essi, mentre, dall'altra, essendo sottoposto anch'esso alle trasformazioni, influenza e travolge le vite dei protagonisti. La complessità della rete dei rapporti che si costruisce in questo modo, tra lo spazio urbano e i residenti, fa uscire alla superficie molteplici problematiche legate all'uomo contemporaneo, nonché fa riflettere sulla tesi che nella letteratura contemporanea l'elemento spaziale sia più presente e più determinante nella vita dei protagonisti rispetto alle coordinate temporali.

In questo intervento utilizzeremo la metodologia ${ }^{3}$ proposta dalla studiosa Hana WirthNesher nel suo studio City Codes. Reading the Modern Urban Novel (1996). Nell'analisi dell'elemento spaziale nei testi narrativi, Wirth-Nesher distingue quattro tipi dell'ambiente urbano: quello "naturale", quello costruito, umano e verbale. ${ }^{4}$ Prima di spiegare cosa sottintenda con ognuno di questi, rileviamo che in questo intervento, parlando dello spazio urbano, utilizzeremo piuttosto il termine 1' 'ambiente', con il quale si sottintende 'l'insieme delle condizioni sociali e culturali in cui una persona vive; l'insieme delle persone che si frequentano, con le quali si è a contatto; l'insieme di persone accomunate da ideali, interessi o attività simili; il complesso di circostanze che rendono possibile il manifestarsi di un fenomeno [...]." Wirth-Nesher separa l'ambiente, questo complesso delle condizioni esterne materiali, sociali, culturali, ecc. nell'ambito delle quali vive, opera ed evolve un essere umano, e lo distingue in quattro categorie: con quello naturale ci si riferisce all' inclusione o intervento

\footnotetext{
${ }^{3}$ Abbiamo già utilizzato la metodologia di Hana Wirth-Nesher in alcune precedenti ricerche che riguardano lo spazio urbano: Vedi, ad esempio, Mihaljević, Nikica. 2018. "Le città moleste: la napoletanità soffocante nei romanzi di Elena Ferrante". La città italiana come spazio letterario nel contesto mediterraneo (1990-2015). Eds. Srećko Jurišić, Antonela Marić, Nikica Mihaljević e Katarina Dalmatin. Firenze: Franco Cesati Editore. 69-78.

${ }^{4}$ Cfr. Wirth-Nesher 1996: 11-13.

${ }^{5}$ Garzanti Linguistica, https://www.garzantilinguistica.it/ricerca/?q=ambiente [27 febbraio 2019] 
della natura nell'ambiente costruito (Cfr. Wirth-Nesher 1996: 11), mentre con quello costruito ci si riferisce alla disposizione geografica di una città, agli oggetti architettonici e ad altri manmade objects come tram, pareti divisorie e tetti (Cfr. Wirth-Nesher 1996: 12). Invece, con l'ambiente umano, il quale riteniamo particolarmente importante nella presente ricerca, ci si riferisce "piuttosto a caratteristiche umane che costituiscono un ambiente, come folle di pendolari, venditori ambulanti e passanti. [...] può facilmente riferirsi a tipi che fanno parte delle caratteristiche generali di una città in determinati periodi o aree: il portiere, il musicista di strada, il mendicante, ecc." (Cfr. Wirth-Nesher 1996: 13). Infine, per l'ambiente verbale sottintende, in un testo narrativo, la lingua scritta e parlata: la prima includerebbe i nomi delle strade e dei luoghi nonché qualsiasi altra lingua che sia visivamente inscritta nel paesaggio urbano - annunci pubblicitari, vari avvisi e graffiti, mentre la seconda si riferisce alla parola pronunciata, sia nella sua espressione colloquiale, dialettale, o qualsiasi altra forma della lingua, o anche più di una, ufficiale (Cfr. Wirth-Nesher 1996: 13-14). Wirth-Nesher sottolinea che, nell'interazione con l'ambiente circostante, vi sarà di fondamentale importanza la percezione dello spazio non solo attraverso la vista, ma anche altri sensi, in modo tale che la polisensorialità avrà un ruolo decisivo in questo interagire tra l'uomo e l'ambiente urbano. ${ }^{6}$

La premessa dalla quale si parte nell'analisi è che, applicando la distinzione tra quattro tipi dell'ambiente nell'analisi di quattro racconti della raccolta Bologna d'autore, due firmati da due autori e due da due autrici, si presuppone di trovarvi la presenza più significativa dell'ambiente costruito, umano e verbale, rispetto a quello naturale. In questo, sarà interessante notare la presenza dei personaggi come l'artista di strada, il senzatetto, il venditore ambulante,

\footnotetext{
${ }^{6}$ Cfr. Wirth-Nesher 1996: 14.
} 
ecc., i quali a volte svolgono le funzioni secondarie rispetto ai personaggi principali, mentre, a volte, sono proprio loro i protagonisti principali. Risulta fondamentale il loro rapporto che, attraverso l'ambiente urbano, si svolge con altri protagonisti e anche con loro stessi. Inoltre, un'attenzione particolare verrà data alle professioni dei protagonisti nel loro rapporto con l'ambiente urbano.

Per confermare ulteriormente l'importanza di questo tipo di metodologia nell'esamina e la descrizione del rapporto tra il personaggio e l'ambiente urbano, occorre spostare l'attenzione su alcuni studi recenti, i quali avvalorano la tesi che oggi gli spazi e i luoghi svolgono una funzione maggiore rispetto al tempo, proprio in quanto, come rileva Marco Maggioli, “[1]'ottica spaziale è considerata da qualche anno ormai, perlomeno a partire da un testo seminale come Third Space del geografo Edward Soja (1996) [...] necessaria per cercare di comprendere e interpretare il presente, oltre che per il rovesciamento interpretativo e prospettico che innesca in un mondo irriducibile ad una superficie piana [...]" (Maggioli 2015: 51). L'autore parla della cosiddetta 'svolta spaziale' (ingl. spatial turn) che ha travolto gli studi letterari, storici, antropologici e culturali in seguito ai cambiamenti sociali. Le discussioni intorno alla 'svolta spaziale' ebbero inizio negli anni ' 80 del secolo scorso, in primo luogo da Bertrand Westphal e altri studiosi, come David Harvey, Edward Soja, Derek Gregory, Steve Pile, Doreen Massey, ecc. Gli studi di geocritica ci rendono coscienti del fatto che la geografia reale e la geografia letteraria, pur essendo dei 'luoghi' diversi, tuttavia si definiscono proprio in base al rapporto che si istaura tra le due, e la 'svolta spaziale' ci fa ragionare proprio sul fatto quanto lo spazio reale sia legato allo spazio letterario, e viceversa. Gli studi riguardo alla 'svolta spaziale' affermano che la geografia, al giorno d'oggi, viene piuttosto percepita come una disciplina interdisciplinare, o, meglio, transdisciplinare, il che afferma l'iniziale ipotesi che la spazialità 
e la territorialità, lo spazio e il territorio, rappresentano degli elementi 'attivi' nell'intersezione tra individuo, luogo e società e che sia l'individuo sia il luogo sia la società sono tutti, nel processo di relazioni, dei soggetti attivi, i quali partecipano, ognuno a modo suo, nella trasformazione dell'altro soggetto facente parte di questo gruppo, influenzandosi a vicenda. Di conseguenza, siamo testimoni dei continui cambiamenti dell'ambiente e della società come anche degli individui stessi, il che ha come risultato una trasformazione continua con molteplici risvolte. Comunque, dato che problematizzare ulteriormente la 'svolta spaziale' non fa parte della presente ricerca, non ci soffermeremo oltre su questa problematica, ${ }^{7}$ ma inizieremo l'analisi riportando le seguenti parole del critico letterario croato Stipe Grgas:

Che si tratti di geografia culturale o antropica, geografia ispirata al marxismo o al femminismo, al centro dell'attenzione vi si trova l'uomo nello spazio, la percezione umana e l'esperienza dello spazio; vengono poste le domande su come lo spazio condizioni la prassi umana, ma anche in che modo gli esseri umani / le società trasformino o creino il mondo circostante. Da queste diverse intersezioni è emersa una nuova geografia che ha riconosciuto la necessità che questa disciplina diventi una pratica integrativa, interdisciplinare e discorsiva. (Grgas 2010: 55) ${ }^{8}$

Riteniamo utile rilevare che, oltre che dallo studio di Wirth-Nesher e la metodologia che propone, anche dalle sottodiscipline, come geografia culturale o antropogeografia, nell'analisi

\footnotetext{
${ }^{7}$ Per una bibliografia più dettagliata riguardo agli studi sulla 'svolta spaziale' e sulle ricerche recenti riguardo alla riqualificazione, nell'ambito degli studi di geografia, della territorialità e del suo valore umano, sociale e storico, rimandiamo ad alcuni testi importanti: Soja, Edward. 1996. Thirdspace: Journeys to Los Angeles and Other Realand-Imagined Places. Oxford: Blackwell Publisher; Weigel, Sigrid. 2001. 'Zum 'topographical turn': Kartographie, Topographie und Raumkonzepte in den Kulturwissenschaften”. KulturPoetik 2, 2: 151-165; Massey, Doreen. 2007. For Space. Los Angeles/London/New Delhi/Singapore: Sage Publications; Günzel, Stephan. 2008. "Spatial turn - topographical turn - topological turn. Über die Unterschiede zwischen Raumparadigmen". Spatial Turn: Das Raumparadigma in den Kultur- und Sozialwissenschaften. Eds. Jörg Döring and Tristan Thielmann. Bielefeld: transcript Verlag. 219-237; Soja, Edward. 2008. "Vom Zeitgeist zum Raumgeist. New Twists on the Spatial Turn". Spatial Turn: Das Raumparadigma in den Kultur- und Sozialwissenschaften. Eds. Jörg Döring and Tristan Thielmann. Bielefeld: transcript Verlag. 241-262; Grgas, Stipe. 2010. "Bučno nadire prostor sa svih strana: Geografija u pjesništvu Adriane Škunca". Muzama iza leđa. Vuković, Tvrko ed. Zagreb: Zagrebačka slavistička škola. 51-72; Westphal, Bertrand. 2011. Geocriticism: Real and Fictional Spaces. New York: Palgrave Macmillan; Brković, Ivana. 2013. "Književni prostori u svjetlu prostornog obrata". Umjetnost riječi LVII, 1-2: 115: 138; Marramao, Giacomo. 2013. "Spatial turn: spazio vissuto e segni dei tempi". Quadranti 1, 1: 31-36; Maggioli, Marco. 2015. "Dentro lo Spatial Turn: luogo e località, spazio e territorio". Semestrale di Studi e Ricerche di Geografia XXVII, 2: 51-66, ecc.
}

${ }^{8} \mathrm{La}$ traduzione dal croato in italiano è dell'autrice dell'intervento. 
delle manifestazioni spaziali, territoriali, geografiche della cultura, dallo studio dei simboli che vengono attribuiti ai luoghi e agli spazi e dell'agire generale dell'uomo e dei fatti dell'uomo avrà l'inizio la presente analisi.

\section{Osservare, annusare, ascoltare e dialogare con una città: lo spazio di Bologna}

La raccolta Bologna d'autore esce nel 2016 e racchiude i racconti firmati da diversi autori. ${ }^{9}$ La raccolta fa parte della collana Città d'autore, edizione di Morellini Editore, a cura di Gabriella Kuruvilla. A partire dal 2014, fino ad oggi sono state pubblicate sei raccolte di racconti: Milano d'autore (2014), Roma d'autore (2015), Monaco d'autore e Bologna d'autore (2016), Genova d'autore (2017) e Calabria d'autore (2018). L'obiettivo degli autori era raccontare varie zone urbane indagando, osservando e cogliendo gli aspetti meno noti e meno caratteristici delle città e regione indicate nei titoli, ovvero gli elementi, i dettagli e gli aspetti meno evidenti, nascosti e sconosciuti, i quali si percepiscono con altri sensori, oltre che con la vista. Nell'analisi, la quale non si soffermerà su tutti i racconti, ma solo quelli nei quali l'ambiente urbano si dimostra particolarmente importante per determinare, condizionare e transformare l'identità dei protagonisti, vedremo come proprio questi aspetti dell'ambiente urbano permettono di raccontare il disagio dell'uomo contemporaneo, l'inquietudine e la vacuità della sua esistenza, nonché altre problematiche nell'interazione con l'ambiente circostante. Inoltre, dato che saranno analizzati due racconti di due autori e due racconti di due autrici, verrà dimostrato che, in questa raccolta, vi è poca differenza tra la scrittura femminile

\footnotetext{
${ }^{9}$ I racconti presenti nella raccolta Bologna d'autore sono firmati dai seguenti autori: Andrea Cotti, Marcello Fois, Otto Gabos, Deborah Gambetta, Gabriella Kuruvilla, Massimo Laganà, Claudio Lolli, Loriano Macchiavelli, Valerio Monteventi, Gianluca Morozzi, Giampiero Rigosi, Gianluca Vittorio.
} 
e quella maschile, quando analizziamo l'individuo e il suo rapporto con l'ambiente urbano. Oltracciò, le differenze stilistiche tra i singoli autori ci faranno scoprire i nuovi mondi, osservati 'dal basso', 'dall'alto', 'da dentro', 'da fuori', 'da vicino' e 'da lontano', costruendo in questo modo 'un prisma urbano' che racconta e mette al centro dell'attenzione l'uomo. D'altra parte, è significativo che proprio Gabriella Kuruvilla ha disegnato le copertine per la collana Città d'autore perché in questo modo, anche se ogni raccolta è diversa dall'altra, alla fine tutte si assomigliano, ovvero il disegno prinicipale, nel quale vengono poi inserite alcune immagini tipiche proprio per quella città o quella regione, ha la stessa 'base'. Questo dettaglio è importante per sottolineare il fatto che, mentre si focalizza la particolarità e l'unicità di una città, in realtà, attraverso questo percorso, si arriva a capire che tutte si assomigliano, perdendo, alla fine, la loro specificità proprio a causa del disagio umano che ha un carattere globale.

La raccolta si apre con il racconto Bologna dall'alto è bellissima di Andrea Cotti in cui il ruolo fondamentale ha l'ambiente umano. Il titolo viene ripreso nella prima frase del racconto, con la quale si descrive il centro della città, ma funziona anche come l'antitesi alla frase che segue subito dopo: "È dal basso che fa schifo" (Cotti 2016). Quest'ultima frase pone l'attenzione su quegli aspetti meno noti e meno evidenti, a prima vista, di una città e fa riflettere sul fatto che ci sia ben altro, oltre al centro storico per il quale la città è famosa. Ed è proprio dell' 'altro' che Andrea Cotti racconta in questo racconto: un uomo, in piedi su un cornicione, che sembra abbia intenzione di suicidarsi, viene fermato dalla polizia. L'agente di polizia Luca $\mathrm{Wu}$, però, sarà il protagonista principale, con le sue 'innate contraddizioni'; come dice lui stesso: "sono nato in Italia, a Bologna, da una famiglia cinese, sono italiano, sono cinese e sono un poliziotto" (Cotti 2016). Dato che l'uomo che Luca Wu ha fermato è un uomo proveniente dal Senegal, arrivato in Italia come clandestino e con l'obbligo di lasciare l'Italia, nel loro 
'incontro' vi troviamo non solo la rappresentazione della società multietnica presente a Bologna, ma soprattutto 'lo scontro' nell'applicare la legge, d'una parte, e la necessità di capire l'altro, dall'altra. Le battute che i due scambiano, evidenziano quanto le storie personali dei due, anche se apparentemente diverse, in fondo si assomigliano: la distanza generazionale è di soli nove anni; sono, tutti e due, e almeno in parte, di origini straniere; tutti e due non appartengono alla classe dei "bolognesi ricchi e borghesi" (Cotti 2016). Tuttavia, il problema non verte intorno all'applicazone della legge sull'immigrazione clandestina o riguardo all'importanza di salvare un uomo dal suicidio, ma fa scoprire il 'vero' problema della società contemporanea: vengono 'denudati' coloro che approfittano degli extracomunitari affittando gli appartamenti 'in nero' e guadagnando le cifre "stellari", mentre le persone senza documenti regolari non hanno scelta che accettare di alloggiarvi. Si deduce che il problema principale della società contemporanea non sono gli immigrati che cercano una vita migliore in Italia, e nemmeno gli italiani di orgine 'ibrida' ma coloro che hanno il potere di approfittare dei primi e dei secondi. Questa stratificazione sociale risulta chiara dal monologo interiore di Luca Wu:

Io li conosco. Anche se sono nato in una famiglia cinese, e ho vissuto nella comunità cinese, sono comunque cresciuto in mezzo agli italiani. [...]

Per gli altri siamo invisibili.

Ma io so chi sono loro.

I bolognesi ricchi e borghesi, con il portafogli gonfio e il macchinone, che votano da sempre a sinistra, che poi hanno votato un sindaco imprenditore di destra, e poi di nuovo hanno votato un sindaco di sinistra che però fa sgomberare i campi nomadi con le ruspe.

Da un po' di tempo questi bolognesi hanno capito che possono fare più soldi ammassando nelle loro seconde o terze case in centro ancora più persone. Persone che pagano come e più degli studenti, e hanno meno pretese. Gli stranieri, gli extracomunitari, i clandestini.

Come in questo appartamento.

I vicini non hanno protestato. [...]

Evidentemente il proprietario è in buoni rapporti con gli altri condomini. Oppure anche gli altri condomini affittano appartamenti in modo simile (Cotti 2016). 
La citazione è particolarmente significativa in quanto sottolinea che l'ambiente urbano è nell'epoca contemporanea sostituito dall'ambiente umano dato che le città sono, in realtà, $\mathrm{i}$ suoi residenti, e perciò la metodologia di Hana Wirth-Nesher, quella che con l'ambiente umano, nel tentativo di raccontare e delineare lo spazio di una città, analizza e racconta i suoi cittadini (regolari o clandestini), la loro origine (italiani, di origini miste e multirazziali, stranieri), le loro professioni e le attività di cui si occupano (agente di polizia, locatore, disoccupato, venditore di fazzoletti di $\operatorname{carta}^{10}$ ), si dimostra del tutto adeguata. Questo aspetto narrativo mette in rilievo un altro problema sociale che va oltre alle differenze sopraelencate tra i personaggi: il traviamento e le sue varie implicazioni, che portano alla completa degenerazione sociale ma anche morale e spirituale degli individui. I veri 'protagonisti' di questa deviazione morale non risultano essere gli stranieri, ma i bolognesi che vi sono nati e che ci abitano e questo fatto aumenta la gravità e la complessità del problema. Dalla citazione trapela anche l'indifferenza che nasce, in altri cittadini, riguardo a questa deviazione sociale e le conseguenze di essa, e l'estremo grado di autoreferenzialità che i 'veri' bolognesi hanno in comune. Quindi, diversamente da un 'comune pensare' che il diverso/lo straniero porti gli squilibri sociali, il racconto mette a fuoco un altro problema, quello delle gerarchie prestabilite, riguardo alle quali nessun cittadino protesta, anzi, pensa soltanto ai propri interessi. Perciò non

\footnotetext{
${ }^{10} \mathrm{Il}$ venditore di fazzoletti di carta è un personaggio che viene spesso riproposto nei racconti di queste raccolte, come, ad esempio, nel racconto Radio Varsavia. Ciò che questo personaggio fa è strettamente legato a ciò che rappresenta nella società italiana contemporanea:

"«Ti serve un accendino? Un pacchetto di fazzoletti?»

Niente. Alla fine almeno i fazzoletti li aveva comprati sul serio. Non valevano niente e si bucavano al primo starnuto" (Cotti 2016). Nella citazione è rappresentata la vacuità del gesto di vendita e dell'acquisto, ma non solo: ancora di più vi viene rilevata la vacuità dell'esistenza di tutti e due i personaggi, che hanno i rapporti superficiali, di interesse, e i quali non vanno oltre il momento in cui si incontrano: il primo è solo interessato a guadagnare qualche soldo, in qualsiasi modo, mentre il secondo è interessato solo a liberarsi di colui che 'invade i suoi spazi'. La simbologia del fazzoletto che 'non vale niente' rimanda all'assenza del valore non solo nei rapporti tra le persone ma anche nella vita in generale.
}

Raudem, Revista de Estudios de las Mujeres. Vol. 6, 2018. ISSN: 2340-9630 
sorprende che la Bologna osservata dal basso sia ripugnante e invivibile, proprio in quanto è cambiata molto recentemente:

«L'Italia è cattiva, e Bologna è cattiva. La gente. Sono tutti arrabbiati. Sono feroci.»

È vero. Chi viene da fuori pensa ai bolognesi allegri, divertenti, che si godono la vita. Invece Bologna è cambiata, e se ci si presta attenzione lo si vede chiaramente. Le persone si muovono con la testa bassa, chiuse, stanche e irritate. Nei negozi i venditori sono bruschi, scostanti. I clacson delle macchine bloccate nel traffico bucano le orecchie. [...] C'è tensione, allarme. Basta poco per far scoppiare una lite (Cotti 2016).

Bologna qui descritta preanuncia la delineazione di un ambiente urbano del tutto nuovo, nel quale l'ambiente umano è centrale, ed è quello intorno al quale gravita la vita dei suoi residenti.

\section{Gli squilibri di Bologna}

Nel racconto Una questione di equilibri di Deborah Gambetta si racconta, invece, un avvenimento passato che ha coinvolto sei protagonisti. Il passato è contrapposto al presente, e il presente ne risente ancora le conseguenze.

Il racconto, costruito con l'analessi frequente, si apre con una breve mail in cui uno dei protagonisti informa altri tre riguardo alla morte improvvisa di un loro amico, proponendo, a questo punto, di incontrarsi tutti a Bologna, lì dove l'uomo è morto. Dato che si tratta di un suicidio inaspettato, il protagonista principale viene travolto dai ricordi legati al suo passato soggiorno nello studentato, insieme con altri quattro protagonisti. Il loro primo incontro 
avvenne proprio nelle vicinanze dello studentato, e questa zona, in particolare l'edificio stesso, avrà un'importanza particolare per tutti e sei i protagonisti. L'edificio dello studentato viene raccontato come un microcosmo che racchiude una realtà complessa e confusionale:

Il viavai continuo per le scale, il telefono che suonava nel sottoscala, gli stereo accesi. Ma soprattutto le feste. Una cosa che aveva fatto a capire era che ogni scusa era buona per festeggiare. Un esame andato bene, un compleanno, una cena che di colpo vedeva riunite decine di persone e allora fuori una chitarra, qualche bottiglia di birra, qualcosa in più da mangiare, magari una pizza fatta in casa o una torta infornata all'ultimo momento. [...]

Mentre stava sdraiato a letto con gli occhi spalancati nel buio incapace di addormentarsi, seguiva la corrente della musica che pulsava all'interno dell'edificio come un cuore che pompava sangue. E quando non era musica erano voci. Timbri differenti che si sovrapponevano, si sommavano, si spezzavano in mezze frasi urlate di cui non riusciva mai a capire il contenuto (Gambetta 2016).

Nella citazione l'edificio viene paragonato ad un corpo con "un cuore che pompava sangue", il che gli attribuisce una specie di autonomia, di indipendenza, simile a quella che caratterizza gli esseri umani. D'altra parte, essendo rappresentato come un microcosmo circoscritto solo a determinate persone, vi si rileva la sua chiusura, l'isolamento e l'autoreferenzialità, caratteristiche per gli individui già descritti nel precedente racconto. Il sovrapporsi delle voci e dei rumori evocano i pensieri intrusivi che si accavallano nella mente di un individuo nella società contemporanea, il quale tende a perdere la propria identità, tra qeuste mille 'voci'. Ma un particolare determinante è che di tutte queste presenze, verbali o mentali, non si riesce a capire il significato, come se la vita stessa degli individui facilmente perdesse il senso. Ed è questo il tema del racconto: il valore e il senso della vita umana. In tal riguardo, il protagonista principale mette in rilievo la distanza e l'assenza di interazione tra l'uomo e l'ambiente urbano in quanto l'uomo non può influenzare certi pensieri né certi avvenimenti, e ancor meno li può gestire, perché certi luoghi sfuggono al suo controllo: "Come 
se quel posto avesse avuto un corpo proprio, fosse stata un'entità a sé stante. Un corpo vivo, indipendente" (Gambetta 2016).

Più avanti si viene a sapere che tutti i protagonisti, a parte Paolo che ha commesso suicidio, erano andati a vivere altrove, volendosi allontanare almeno fisicamente da Bologna, quanto più possibile. Il distacco da quell'ambiente, quindi, è stato voluto e necessario, anche se il passato torna e ora Bologna di nuovo assume l'importanza nell'avvicinarli. Allo stesso modo li aveva avvicinati prima, all'epoca quando erano studenti, ed ora ci si interroga sulla 'resa dei conti': “[f]orse era solo un modo per saldare un debito, l'elastico teso all'estremo che ora invertiva la corsa. La linea tracciata venticinque anni prima che ora si ripiegava e chiudeva il cerchio" (Gambetta 2016). Però, Mattia, il protagonista principale, a questo punto, offre gli indizi che proprio quell'ambiente abbia determinato le vite dei personaggi sopraccitati, portandosi via anche una vittima, colui che “[1]'unico che invece era rimasto. L'unico che una mattina si era svegliato e aveva deciso di farla finita. L'unico che aveva pagato - forse - per quello che avevano commesso" (Gambetta 2016).

In questo racconto, bisogna rilevarlo, si focalizza l'importanza di un luogo in particolare, il quale nella società contemporanea, come suggeriscono i racconti della collana Città d'autore, subentra al posto della casa, prendendo la sua, ormai passata, funzione: il bar e/o il ristorante. ${ }^{11}$ Quello che incita l'uomo a frequentare e soggiornare nei bar più che a casa è la sua riscoperta

\footnotetext{
${ }^{11}$ In un'altra sua opera Gabriella Kuruvilla scrive: "Il mio bar assomiglia alla mia panchina che assomiglia al mio palazzo" (Kuruvilla 2012: 6-7). I bar vengono frequentemente evocati nelle opere di Kuruvilla, come i non luoghi o le eterotopie, i quali hanno sostituito le case. Sono i punti di ritrovo, dove le persone si rifugiano, per perdersi nell'alienazione. Anche nel racconto Aldiqua, dalla raccolta Milano d'autore, Kuruvilla riprende lo stesso tema, il bar che è subentrato, come il luogo intimo, alla casa: "In quel periodo, tu e Michele stavate ristrutturando un ex spazio industriale-commerciale, [...]. Per trasformarlo nel Frida: uno dei primi locali alternativi ai bar e alle trattorie, spesso a conduzione e ad atmosfera familiare, della zona. Che, quasi subito, è diventato un punto di ritrovo, molto frequentato [...]" (Kuruvilla 2014).
} 
familiarità ed accoglienza. Questi luoghi diventano i 'luoghi addomesticati', i quali hanno le prerogative di poter offrire, alle persone che vi entrano, le emozioni una volta circoscritte soltanto alle case. Tuttavia, in seguito a ciò, le case diventano luoghi detronizzati, in quanto, nella società contemporanea, hanno perso la loro caratteristica intrinseca, quella dell'intimità e dell'isolamento, nonché di protezione. Perciò l'individuo si trova davanti alla necessità di dover cercare altri luoghi nei quali ritrovare queste caratteristiche. Quindi, come luogosostituto della casa vengono 'scoperti' il bar e il ristorante, i quali hanno una doppia valenza: d'una parte, offrono la possibilità all'individuo di incontrare altre persone, di socializzare, dialogare e confidarsi, mentre, dall'altra, sono anche le zone di passaggio, le quali permettono di mantenere la distanza da altre persone e di avere i rapporti superficiali. È vero che in quei luoghi l'uomo cerca soprattutto l'accoglienza, nonché di star bene, ma è altrettanto vero che questi stessi luoghi gli offrono il disimpegno: non deve né fare niente, né parlare, né partecipare in nessun modo a quello che altre persone che vi sono presenti fanno. La parola chiave è, quindi, il disimpegno, che rappresenta una novità rispetto alle caratteristiche tipiche di una casa; a casa, con altre persone che vi abitano, risulta difficile potersi disimpegnare, a causa del legame familiare che vi è inevitabilmente presente e che l'uomo contemporaneo sembra non sopportare più.

Anche nel racconto Una questione di equilibri il ristorante è un luogo di passaggio o, meglio, un'eterotopia in quanto rappresenta lo spazio che neutralizza e compensa lo spazio di una casa. Prendendo in esempio una stanza in particolare all'interno di una casa, cioè, la cucina, si può determinare che le sue funzioni sono la preparazione e la consumazione del cibo, nonché, durante i pasti, l'interazione con altri che risiedono in quella casa. Questa sua caratteristica è fondamentale per la vita psichica degli individui, non solo in quanto utile per la soddisfazione 
dei loro bisogni primari. Il momento in cui questi bisogni vengono soddisfatti in altri luoghi, fuori casa, ad esempio, nei ristoranti, la casa perde alcune sue caratteristiche, mentre il ristorante acquista altre, nuove. Ad esempio, in questo racconto, i due protagonisti, che si incontrano a Bologna dopo la notizia della morte dell'amico, si avviano proprio verso un ristorante per ritrovare l'intimità del rapporto avuto anni prima:

Si siedono a un tavolo di un ristorante scelto a caso dietro piazza Maggiore, il primo in cui si imbattono. Non conoscono più niente di Bologna, e forse non l'hanno mai conosciuta. [...] Ordinano un misto di salumi, formaggi, tigelle, una bottiglia di vino bianco e spilluzzicano grissini in attesa che arrivino le portate. Prima o poi il momento di spaccare il frutto e affondare le mani ad afferrare il nocciolo arriverà, ma per ora ancora no. Ci si avvicinano per movimenti lenti, cerchi concentrici.

Mattia versa il vino [...].

«Dimmi di Paolo. Come 1'hai saputo?» (Gambetta 2016).

È da notare che la scarsa conoscenza della città sembra essere legata alla stentata e frammentaria dimestichezza tra i due uomini; questi stanno affrontando e rievocando un momento particolarmente importante del passato comune, che ha profondamente condizionato la loro e la vita di altri personaggi, e lo fanno in un luogo di passaggio, fino a un certo punto anche anonimo, dato che proprio questo luogo garantirà a loro di affrontare alcuni argomenti seri e profondi ma, allo stesso tempo, di 'rimanerne fuori', ovvero di mantenere il disimpegno, la via di fuga. In effetti, un discorso del genere non potrebbe, viste le condizioni e il coinvolgimento dei personaggi, svolgersi in uno spazio diverso da quello.

Un ambiente simile viene usato come elemento spaziale in un altro racconto di questa raccolta, Io e l'altra, di Gabriella Kuruvilla, dove la protagonista del racconto è Viola, una 
donna quasi cinquantenne, che ha una tormentata relazione amorosa con una giovane ragazza africana. Il racconto si apre con l'incontro tra le due donne che avviene proprio in un bar:

«Lo sapevo che prenderci una birra, per chiacchierare un po', prima di andare a ballare, era una pessima idea.

La pessima idea stava in quell'inciso: "per chiacchierare un po' ".

La nostra relazione, che già arranca, se si sposta sui binari del dialogo, deraglia. Quantomeno.

Per fortuna, nel bar che ho scelto, il volume della musica è così alto che il massimo della comunicazione che riusciamo a instaurare tra noi è l'urlarci, a vicenda: "Cosa hai detto?"» (Kuruvilla 2016)

La citazione richiama di nuovo la caratteristica principale dei luoghi come i bar di offrire alle persone, in un certo senso, uno spazio intimo e un ambiente accogliente, nonché, allo stesso tempo, fa ragionare sul bisogno dell'uomo di, contemporaneamente, soddisfare il bisogno di interagire con l'altro, in contraddizione con la sua necessità di stare da solo, di vivere senza impegni. L'ambiente di un bar determina la vita delle due donne perché 'non permette' loro di poter parlare liberamente, a causa della musica ad alto volume, ma, d'altra parte, concede la via di fuga, poter prendersi una pausa dall'altro e disimpegnarsi. In questo senso il bar è un'eterotopia molto significativa, e, conseguentemente, non sorprende che, ad esempio, l'ora dell'aperitivo, il momento in cui ci si da' l'appuntamento al bar, diventi un'ora di riferimento, decisiva per certe scelte o ragionamenti, in quanto i protagonisti, durante la pausa dell'aperitivo, 'misurano' i rapporti che hanno, anzi, organizzano le loro vite in base a questo tipo di appuntamento che ha del tutto sostituito il pranzo o la cena di una volta quando la famiglia si riuniva a casa. È significativo anche che la protagonista di Io e l'altra dice alla giovane amante che tornerà a casa proprio all'ora dell'aperitivo; una volta rientrata proprio a quell'ora, le due si lasciano e il loro rapporto finisce. 
Ritornando all'importanza dell'edificio dello studentato, nel quale ha avuto luogo l'avvenimento che ha causato il suicidio di uno dei protagonisti, poniamo l'attenzione al fatto che Leonardo, l'uomo con il quale il protagonista principale scambia i ragionamenti e i ricordi, rimanda l'importanza ad alcuni dettagli non secondari: lui rileva che in quell'edificio "le cose seguivano una logica diversa. Non era come stare in un appartamento normale. Le cose che funzionavano fuori, in qualunque altro posto, là diventavano altre. Era così, gli studentati erano così. Erano altro" (Gambetta 2016). Il riferimento ad un luogo 'altro' fa pensare ad un non luogo, ovvero ad un luogo anonimo, costruito per un fine ben specifico, ovvero per offrire il soggiorno temporaneo agli studenti. In questo senso, lo studentato rappresenta una zona di passaggio, di transito, nella quale si soggiorna solo temporaneamente. D'altra parte, le persone che vi soggiornano tuttavia stabiliscono i rapporti con quei luoghi, pur non essendo i luoghi identitari e nonostante i personaggi che vi soggiornano siano dei semplici utenti di questi luoghi. Questi luoghi, inoltre, hanno una caratteristica particolare: influenzano coloro che vi soggiornano in modo tale che essi si riducano ad essere solo clienti o utenti degli stessi, senza poter far avvalorare le loro caratteristiche personali, né realizzarle o crescere e migliorare dal punto di vista del carattere o di evolvere la capacità di ragionamento. Perciò non sorprende che il fatto avvenuto nel passato sia ambientato proprio in un non luogo e che i protagonisti sentano il bisogno di soggiornare in questi luoghi e di tornarvi, perché:

Là non c'erano appartamenti privati, c'erano solo studenti, più di cento individui della stessa età, più di cento stili di vita differenti, più di cento persone differenti che si influenzavano a vicenda, che vivevano insieme ventiquattr'ore su ventiquattro, rinchiusi dentro lo stesso edificio. Là non c'erano norme, di nessun tipo, niente confronti inevitabili con la famigliola del piano di sotto, niente lamentele, niente rischi di essere cacciati e, soprattutto, niente controlli. Era diverso. Le norme erano modellate sui piaceri. Quello, lo studentato, era un'oasi, una comunità chiusa che delle regole, dei genitori e delle convenzioni finalmente ne poteva fare a meno. Era un'isola. Una scheggia di società ideale che col mondo di fuori non aveva nulla a che fare. Eravamo noi a comandare. È questo il punto. (Gambetta 2016) 
La citazione è significativa per vari motivi. In primis, descrive un edificio come un'isola, una zona separata dal resto del mondo, nella quale tutto è concesso e le regole della vita reale, che rimane esterna, non vi valgono. Nel testo lo stesso ambiente viene definito come un ambiente perfetto per una società ideale, anche se, comunque, limitata dal tempo e dallo spazio, quindi, con le libertà circoscritte. D'una parte, il soggiorno in questi luoghi fa ragionare sul fatto quanto l'uomo contemporaneo abbia bisogno dei piaceri e di soddisfare gli stessi, nonché quanto la sua vita sia condizionata da essi, a tal punto che si lascia governare e determinare dal piacere instantaneo. Dall'altra, lo stesso edificio fa riflettere sul fatto quanto, nella società contemporanea, sia presente il bisogno di evitare i problemi, le autorità, le regole e le convenzioni in generale, le quali sembrano essere arrivate al punto di non poter esser più sopportate. Ma proprio questa caratteristica dell'uomo contemporaneo che non sa controllare il proprio bisogno dei piaceri si dimostrerà fatale in quanto, più avanti, si scoprirà che nello studentato fu uccisa una ragazza, in seguito all'abuso di alcol e di droghe dalla parte dei protagonisti, i quali, di conseguenza, non erano lucidi nel momento quando l'avevano spinta giù dal balcone. La tragedia che ora è svelata sembra mettere in rilievo 'l'ammonizione' all'uomo contemporaneo di tenere a mente in quale direzione possa 'andare' la sua vita trasgredendo le leggi di una collettività e che i piaceri, i bisogni e i sentimenti non possono che essere tenuti sotto controllo, soprattutto nel contesto della vita in collettività. Ciò contrasta con la società contemporanea la quale, essendo fluida, tende a rivedere in continuazione le proprie regole. Inoltre, l'accaduto fa ragionare sul fatto fino a che punto ci si possa lasciar andare ai propri bisogni e desideri o fino a che punto vadano tenuti sotto controllo gli stessi, in una società che sempre di più trasgredisce le regole e tende a spostare i limiti e a superarli. In più, il fatto fa riflettere su come lo spazio delimitato influenzi comunque coloro che vi soggiornano, anche se i residenti di esso siano dei semplici utenti, cambiando le loro abitudini, i loro modi 
di comportamento e i loro atti. Quindi, l'influenza che questi luoghi esercitano sulle persone, cambiandole in peggio e facendo trasgredire le regole stabilite in una società, rendono il comportamento dell'individuo, quando vi soggiorna, imprevedibile. I non luoghi del genere risultano essere inevitabili ma allo stesso tempo sono una malattia della società contemporanea, un male ineluttabile.

\section{E se la trasformazione dei luoghi urbani fosse determinante per i protagonisti?}

Nell'epoca contemporanea caratterizzata dall'insicurezza, precarietà ed imprevedibilità, l'intervento nell'ambiente urbano, in particolare riguardo alle zone in degrado, rispecchia il bisogno dell'uomo contemporaneo di cambiare ciò che succede intorno a lui ovvero di interventire nella vita collettiva. Così si ha un'interazione tra l'ambiente costruito e l'ambiente umano, in quanto l'uomo si impegna di salvare certe zone dal degrado e di contribuire alla collettività, trasformando questi luoghi e modificandone la funzione; tra questi possiamo citare, ad esempio, i centri sociali, i quali, evidentemente, nel frattempo hanno perso la loro funzione primaria, oppure gli ex complessi industriali. Queste trasformazioni rispecchiano la necessità dell'uomo di intervenire nelle problematiche sociali contemporanee, con lo scopo di migliorare, fondamentalmente, la vita a se stesso. Nel racconto Io e l'altra, un luogo del genere è la via Stalingrado, in periferia di Bologna. La voce narrante rileva:

Deve esserci un'attrazione che persiste verso questi spazi in disuso, degradati e fatiscenti, abbandonati da anni e destinati alla demolizione. Che vengono occupati, da alcune persone e dalle loro iniziative, temporaneamente. $\mathrm{E}$ che, anche geograficamente, rispetto ai confini della città, non si trovano dentro e neppure fuori. Sono ai margini, in bilico, sospesi e precari. Come mi sentivo e mi sento, spesso. Pure questa percezione di me persiste. Poi però ci sono dettagli, che evidenziano le differenze: solo la definizione del luogo e il nome della strada sono uguali. Il 
posto no. "Tutto cambia" è una frase che ho sempre vissuto come un epitaffio, e con cui ho lottato come Don Chisciotte contro i mulini a vento (Kuruvilla 2016).

La voce narrante paragona se stessa con i cambiamenti che travolgono la città evidenziando quanto tutto sia soggetto ai cambiamenti e proprio ciò è uno dei problemi dell'epoca contemporanea: la vita dell'uomo, anche contrariamente alla volontà di lui, cambia, e, spesso, deteriora, ma, è ancora più importante sottolineare che questi luoghi si trovano né dentro né fuori dei confini della città, "ai margini, in bilico, sospesi e precari”, come lo è anche la vita umana e come lo sono altri non luoghi menzionati precedentemente.

In questo racconto è importante porre l'attenzione alla rappresentazione dell'ambiente verbale: la voce narrante racconta che un ex centro sociale è stato trasformato in "un museo all'aperto gratuito" dove oggi ci sono i murales. Questi ultimi, essendo l'espressione, sul tessuto urbano, attraverso le parole, di idee, atteggiamenti ed emozioni di un individuo o un gruppo, sono chiamati da Wirth-Nesher l'ambiente verbale, in quanto le parole scritte costruiscono e modificano allo stesso tempo l'ambiente urbano. Quindi, con la presenza dei murales, si ha la compresenza dell'ambiente costruito, quello umano e quello verbale, i quali sono tutti e tre l'espressione dello stato in cui si trova l'uomo di oggi, ma è altrettanto importante che questi tre tipi dell'ambiente si influenzano a vicenda.

Nella rappresentazione di Bologna in questo racconto la via Stalingrado è importante in quanto un altro non luogo:

[...] più che una via è un vialone, a due corsie, diviso da uno spartitraffico e circondato da caserme e fabbriche dismesse, tra cui sono sorti anonimi edifici da terziario avanzato. Praticamente è un raccordo, tra la tangenziale e la città, sospeso tra passato e presente, come qualcosa che non sta né di qua né di là. Qualcosa di non riuscito, che potrebbe trovarsi ovunque. Un non luogo qualsiasi (Kuruvilla 2016). 
Oltre ad essere la testimonianza del passaggio dei veicoli e delle persone, la via è il simbolo di una zona in-between, sia geograficamente sia per quanto riguarda l'ambiente urbano e quello umano. Ciò significa che questa via è soprattutto importante per l'uomo perché, in realtà, è l'uomo contemporaneo che si sente di essere costantemente in-between, né di qua né di la, nei rapporti che vive con altri, ma anche con se stesso. Perciò, in realtà, partendo da questa sua caratteristica, l'uomo stesso risulta essere un non luogo. Inoltre, la voce narrante ricorda la canzone degli Stormy Six, i quali cantavano la via Stalingrado come il simbolo della liberazione dai nazisti. Paradossalmente, però, ora questa via non rappresenta più il simbolo della libertà, ma, anzi, proprio l'opposto, una strada “[c]he serve, più che altro, al traffico e al commercio: alle macchine, alle merci e alle loro necessità. Dunque: omaggia bisogni e dipendenze, invece di inneggiare alla liberazione e alla libertà" (Kuruvilla 2016). Al posto della liberazione è subentrata un'altra caratteristica tipica per la società contemporanea e per l'uomo di oggi: la dipendenza, forse la parola che descrive l'uomo di oggi nel migliore dei modi, il quale passa da una dipendenza all'altra per riempire il vuoto interiore. Inoltre, questi richiami del passato, attraverso i paragoni tra quello che c'è stato e quello che c'è ora, inneggiano anche al bisogno dell'uomo di rimanere attaccato alla propria giovinezza. Perciò non sorprende che i luoghi prediletti dei personaggi sono i luoghi dove si va a ballare ${ }^{12}$, come le discoteche, in quanto queste permettono di azzerare il proprio presente e passato, ma anche di annullare la propria identità. Kuruvilla sottolinea: "Diventavamo puro movimento, anestetizzandoci: era meno faticoso che andare a correre e più sano che drogarsi. Comunque, qualcosa di molto

\footnotetext{
${ }^{12}$ Anche in altri racconti di Kuruvilla, Aldiqua o G8, nei quali si descrivono altre città, come Milano e Genova, i protagonisti vanno spesso a ballare nei luoghi dove si ha una maggiore interattività come ad es. nelle dancehall, ex complessi industriali trasformati in bar o locali, oppure ai concerti, $i$ quali, contemporaneamente, garantiscono anche il disimpegno.
}

Raudem, Revista de Estudios de las Mujeres. Vol. 6, 2018. ISSN: 2340-9630 
simile alla felicità" (Kuruvilla 2016). Quindi, si tratta degli ambienti che danno all'uomo la possibilità, senza dover rovinare la propria salute drogandosi o ubriacandosi, di godere dello stesso piacere: la serenità, anche se temporanea, che oramai l'uomo contemporaneo non riesce più a trovare in altri modi e luoghi.

\section{Il malessere esistenziale e l'inquietudine raccontati attraverso l'ambiente umano}

Nel racconto Lo strillone della libertà di Valerio Monteventi troveremo l'esempio dell'ambiente umano che appare nettamente predominante rispetto ad altri tipi di ambiente urbano.

La voce narrante in questo racconto è un senzatetto, il quale racconta la propria visione della città: "Se mi chiedete qual è stato il mio primo impatto con Bologna, vi rispondo: orizzontale. [...] Questa sarà pure la città delle torri, ma il numero dei tacchi a spillo che ho visto nei giorni che ho vissuto disteso sui cartoni è stato da record mondiale. [...] Arrancavo dal basso verso l'alto per cercare di sopravvivere..." (Monteventi 2016). La descrizione della città dalla parte della voce narrante è del tutto diversa rispetto alle rappresentazioni presenti in altri racconti precedentemente analizzati; in questo caso la città viene osservata dal basso verso l'alto. Per il protagonista, i portici non rappresentano una zona da visitare, come per i turisti, ma per lui svolgono un'altra funzione, quella "di proteggere dalle intemperie.” La Bologna raccontata qui è una città che offre il rifugio e la protezione al protagonista, a differenza da quella descritta in Bologna dall'alto è bellissima: "Rimanere nella stessa zona ti fa conoscere 
qualche persona di buona volontà che trova piacere nel protarti un aiutino quotidiano o nello scambiare una parola. Inoltre, la stanzialità di far entrare nell'inventario delle associazioni che aiutano i clochard [...] puoi sperare in una presa in carico dei servizi sociali” (Monteventi 2016). Tuttavia, non mancano neanche coloro che, camminando, gli allungano una pedata, così che il protagonista spesso deve cercare un altro rifugio. Dal punto di vista di un senzatetto, si viene a conoscere un'altra Bologna, quellla delle regole della vita di strada.

In questo racconto un dettaglio interessante rappresenta "Piazza Grande", "il primo giornale di strada, scritto e distribuito dai clochard" (Monteventi 2016). In seguito, questo nome/titolo e iniziativa si sviluppano e trasformano in un'associazione formata dai volontari, degli ex clochard, i quali distribuivano il cibo, le bevande e le coperte ad altri senzatetto. Vengono raccontati, in questo modo, altri abitanti di Bologna, i quali sono dei veri e propri inbetweeners, e perciò non sorprende che soggiornano nei luoghi eterotopici, come le biblioteche, i quali non solo fanno vedere come utilizzare alcuni luoghi, predestinati ad altro, adeguandole ad altri bisogni e alle nuove esigenze, ma fanno rilevare anche altre problematiche della società contemporanea, come, ad esempio, la questione riguardo ai bagni publici i quali rivelano lo stato del progresso culturale di un paese, e qui sono il motivo per cui, tra l'altro, la voce narrante si rifugia in una biblioteca. Anche se pure in questo caso i bar e le osterie saranno i luoghi frequentati dai clochard, qui hanno un'altra funzione rispetto a quella descritta nei racconti precedenti: diventano le zone nelle quali i senzatetto possono sperare di ottenere un piatto gratuito o di, magari, trovare un lavoro temporaneo, quindi, sono le zone che stabiliscono la reciprocità dei rapporti. Anche questo fatto conferma che i bar diventano i luoghi di cordialità e accoglienza, più delle case. 
Come detto prima, in questo racconto un'attenzione particolare viene data ai bagni pubblici, i quali fanno parte dell'ambiente urbano, raccontando una Bologna particolare. È significativo che gli orari dell'apertura e di chiusura dei bagni pubblici sono legati all'ora dell' aperitivo, perché la loro chiusura avviene proprio a quell'ora. In questo modo, possiamo stabilire una relazione tra i protagonisti dei precedenti racconti i quali iniziavano, mantenevano e portavano a termine i loro rapporti con altri personaggi proprio all'ora dell'aperitivo, mentre in questo caso l'ora dell'aperitivo è legata ad un bisogno primario, quando i bagni pubblici vengono chiusi, condizionando la vita dei senzatetto. Questo aspetto pone l'attenzione sul fatto che per l'uomo contemporaneo scompare la differenza tra il bisogno primario e il bisogno di avere relazioni sociali, ma, contemporaneamente, rimane la sua necessità di mantenere la possibilità del disimpegno da tutti e da tutto. Perciò, un senzatetto ne è un esempio significativo: vi è al centro dell'attenzione un individuo privo di un'abitazione o di un alloggio, il quale non ha legami stretti con uno spazio privato e intimo. Anche altri spazi eterotopici, come il cinema, o il caffè letterario, accanto alla già menzionata biblioteca, spostano l'attenzione verso un mondo del tutto sconosciuto, pur sempre dei residenti di Bologna, quello di coloro che non hanno una casa e vivono per strada. È chiaro che nel racconto si vuole porre l'attenzione su un'altra problematica sociale, cioè il modo in cui le persone senza casa vengono trattate, evidenziando la loro presenza nelle città: “Un tempo l'obiettivo era di fare la guerra alla povertà, adesso la fanno ai poveri", si indica nel racconto. "Vogliono mettere la miseria sotto lo zerbino, prendendosela con mendicanti o vucumprà. Fanno retate contro i lavavetri come se fossero componenti di una pericolosa cosca mafiosa" (Monteventi 2016). In questa frase è racchiuso uno dei problemi principali della società contemporanea, che non focalizza e risolve i problemi veri, ma sposta l'attenzione dei suoi residenti e delle autorità verso coloro che in realtà andrebbero aiutati in modo adeguato e non perseguiti dalla polizia. In questo senso, 
anche il presente racconto affronta un argomento complesso: raccontando l'ambiente urbano in realtà si racconta soltanto l'ambiente umano.

\section{Conclusione}

Analizzando quattro racconti della raccolta Bologna d'autore, con la metodologia di Hana Wirth-Nesher, siamo arrivati alla conclusione che il tipo dell'ambiente che vi predomina è quello umano, in primo luogo, seguito da quello costruito e quello verbale, senza distinzione se si tratti della scrittura femminile o quella maschile. Da ciò deduciamo che la città contemporanea fa di sfondo alla vita degli individui, e quindi l'interazione, ovvero la reciproca influenza, tra l'ambiente umano e quello costruito, è molto presente nei racconti analizzati. Tuuttavia, questi racconti testimoniano com'è cambiato il modo di vivere l'ambiente urbano e come sempre più spesso i luoghi e le zone urbane acquistano una nuova funzione: soddisfare i piaceri dell'uomo, e non solo i suoi bisogni primari, ma di più quelli sociali, facendogli credere che, in questo modo, lo stanno aiutando a realizzare la propria identità. In seguito all' estraniazione, che ha caratterizzato tutto il '900 letterario italiano, ora sembra riapparire il bisogno di autorealizzazione attraverso il 'bisogno sociale', ovvero il 'bisogno di una società', anche se questo bisogno non è ancora chiaramente individuato né espresso. Però, ciò su cui la letteratura contemporanea italiana pone l'attenzione è il fatto che comunque, in un futuro non lontano, probabilmente ci sarà sempre più crescente la tendenza di sviluppo della ricerca del modo per soddisfare i bisogni non dei singoli individui ma di un sistema di bisogni generale, che va al di là dei bisogni personali. E l'ambiente urbano, come confermato nei racconti 
analizzati, va adeguato a questi bisogni. Forse questi quattro racconti fanno riflettere sul fatto che ciò stia già avvenendolo, $\mathrm{o}$, se no, che sia arrivato il momento di affrontare questo problema con più attenzione.

\section{Riferimenti bibliografici}

Cotti, Andrea. Bologna dall'alto è bellissima. 2016. In Kuruvilla, Gabriella, ed. Bologna d'autore. Milano: Morellini Editore. E-book.

Gambetta, Deborah. Una questione di equilibri. 2016. In Kuruvilla, Gabriella, ed. Bologna d'autore. Milano: Morellini Editore. E-book.

Grgas, Stipe. 2010. "Bučno nadire prostor sa svih strana: Geografija u pjesništvu Adriane Škunca”. Muzama iza leđa. Vuković, Tvrko ed. Zagreb: Zagrebačka slavistička škola. $51-72$.

Kuruvilla, Gabriella. 2012. Milano fin qui tutto bene. Milano: Laterza - Contromano. . 2014. Milano d'autore. Milano: Morellini Editore. E-book. . (ed). 2016. Bologna d'autore. Milano: Morellini Editore. E-book. . Io e l'altra. 2016. In Kuruvilla, Gabriella, Bologna d'autore. Milano: Morellini Editore. E-book.

Monteventi, Valerio. 2016. Lo strillone della libertà. In Kuruvilla, Gabriella, Bologna d'autore. Milano: Morellini Editore. E-book.

Wirth-Nesher, Hana. 1996. City Codes. Reading the Modern Urban Novel. Cambridge: Cambridge University Press.

\section{Siti internet}

Garzanti Linguistica, https://www.garzantilinguistica.it/ricerca/?q=ambiente [27 febbraio 2019] 